\title{
Screening effects through electron-electron interaction in the crystal structures of an intermediate dimension
}

\author{
N.K. Tovstyuk \\ Ivan Franko Lviv National University, Department of Electronics \\ 50, Dragomanova str., 29005 Lviv, Ukraine \\ E-mail: tose@polynet.lviv.ua
}

Abstract. In the paper studied are polarization properties of an electron subsystem in the structures of intermediate dimension, particularly, in the quasi-two-dimensional structures depending on the degree of quasi-two-dimensionality. These investigations were founded on the model dispersion law that most completely governs the peculiarities of the structure. Here, we studied the character of alternating oscillations of the potential screened by interelectron interaction, namely, by its anisotropy depending on microscopic parameters that determine the shape of isoenergetic surface and the degree of quasi-twodimensionality. It is shown that the obtained potential, in comparison with similar results for isotropic Fermi gas, oscillates at smaller distances with amplitude of an order of magnitude higher and with a period of an order of that smaller.

Keywords: quasi-two-dimensional structure, interelectron interaction, polarization properties, singuliarity points.

Manuscript received: 18.03.05; accepted for publication 18.05.05.

\section{Introduction}

The recently subject of intensive studies are lowdimensional structures, i.e., quantum dots, onedimensional (1D) wire and two-dimensional (2D) structures [1, 2]. Such investigations are not only of fundamental interest, but also are more important for the applications of these objects. A number of precise results of the studies of such objects are well known [3, 4]. However, the structures which can be considered as intermediate structures between zero- and $1 \mathrm{D}$, or $1 \mathrm{D}$ and $2 \mathrm{D}, 2 \mathrm{D}$ and $3 \mathrm{D}$ ones have been realized [5], the latter including the layer crystals. The layer crystal can be represented as a set of packed "sandwiches" bonded by weak van der Waals forces. Each sandwich is a set of monoatomic layers with covalent or ion-covalent bond. Such discrimination in chemical bonds causes a number of phenomena typical for the layer crystals. The degree of $2 \mathrm{D}$ or $3 \mathrm{D}$ can be significantly changed by different ways, such as a pressure hydrostatic or axial (along the normal to the layers) or intercalation [6]. In other words, the layer crystals can be considered as a quasi 2D structure (Q2D), or, in the case of decreased interlayer binding, we can consider it as the system of $2 \mathrm{D}$ structures weakly bonded to one another. It is known that electron mixing at the sites of a crystal determines the width of the conduction band. Hence, the difference of the value of mixing inside the layer and between the layers is the reason why the bandwidth in the layer is much higher than that between the layers in the reciprocal space. It demands to refuse from the widely used representation of the dispersion law of carriers by the parabolic dependence, which is possible for a wide band (i.e., within the layer), but is not valid for a narrow band. In the latter case, even at a very low degree of band occupancy, the deviation from the parabolic dispersion law can be significant. It means that the peculiarities of a layer crystal manifest themselves at the stage of its one particle studies, which should be all the more so taken into consideration in the case of many-body effects.

The aim of this work is

- to analyze some many-body characteristics depending on the degree of "quasi-two-dimensionality" of the system on the assumption of a model dispersion law, that most completely governs the structure peculiarities of the crystal of an intermediate dimension;

- to study how the dimension of crystal structure including the nonparabolic band structure (in those cases when the effective mass approximation is forbidden) affects the polarization properties of the electron subsystem.

2. Model dispersion law and its application to the description of many-particle effects

Let us consider the following model dispersion law (in the case when $O Z$ axis coincides with the normal to the layers) [7] 
$E\left(k_{x}, k_{y}, k_{z}\right)=\alpha\left(k_{x}^{2}+k_{y}^{2}\right)+t\left(1-\cos k_{z}\right)$

where $\alpha=\frac{1}{2 m_{x, y}^{*}} \quad\left(m_{x, y}^{*}\right.$ is the isotropic effective mass of an electron inside the layer), $t$ is an integral of electron mixing between the nearest layers along the normal to them, which determines the conduction bandwidth, $2 t$, in the $O Z$ direction of the reciprocal space. The lattice constants and $\hbar$ were chosen to be equal to unity.

The discontinuity of the ion structure is considered as the discontinuity of the value of wavevectors $k_{x} k_{y}, k_{z}$, that is caused by the Born - Karman conditions. The choice of the effective mass approximation for the description of electrons inside the $X O Y$ layer and the tight binding across the layers is imposed by the abovementioned significant difference of chemical bondings in various $O X, O Y$, and $O Z$ crystallographical directions of the layer crystal, which causes the condition $2 t<<\pi^{2} \alpha$, i.e., the case of the intermediate dimension.

The model dispersion law (1) is convenient for the mentioned problem solution, inasmuch as the change of $t$ parameter makes it possible to realize the $2 \mathrm{D}$ structure in the $t=0$ limit. When $t$ increases $\left(2 t \rightarrow \pi^{2} \alpha\right)$ the nonparabolic effects along $k_{z}$ can be neglected, and then we take this 3D anisotropic parabolic dispersion law. The limit value of the parameter $\alpha=0$ describes the 1D structure. The model dispersion law (1) causes the phase $2 \frac{1}{2}$ Lifshitz transition, i.e., topological changes of isoenergetic surfaces according to the energy or chemical potential changes, which should manifest themselves in polarization properties of layer crystals.

It is clear that interpretating such crystals as the system with an intermediate dimension, their physical properties, in particular, polarization ones should be compared to the properties of pure 2D (when $t=0$ ) or 3D structures (when $2 t \rightarrow \pi^{2} \alpha$ ). However, the smooth transition from $2 \mathrm{D}$ to $3 \mathrm{D}$ case for $t<\alpha$ may not be observed.

Many-body effects in the electron subsystem of the crystal with intermediate dimension can have a specific character due to the peculiarities of the band structure. For example, 1D system studies [8, 9] using the dispersion law (1) in the case $\alpha=0$ showed an additive singularity of the dielectric function when $q_{z}=2\left(\pi-k_{\mathrm{F}}\right)$.

The Fourier form of the screened Coulomb potential related with the core potential $V_{0}(\mathbf{q})$ through the wellknown expression [10] that, in the $\Omega=0$ case, is as follows:

$\tilde{V}(\mathbf{q})=\frac{V_{0}(\mathbf{q})}{\varepsilon(\mathbf{q})}$,

where $V_{0}(\mathbf{q})$ for 3D structures has the form [10]
$V_{0}(\mathbf{q})=\frac{4 \pi e^{2}}{\varepsilon_{0}|\mathbf{q}|^{2}}$

and $\delta(\mathbf{q})$ is the dielectric function. We will be interested in the behaviour of the potential screened by electron-electron interaction for systems with an intermediate dimension which is inspected for anisotropy of the conduction band. That is why both in the $t<\alpha$ case and in the limit cases $t \rightarrow 0, t \rightarrow \pi^{2} \alpha$ the Coulomb potential will be given as (3).

Statistic description of many-body effects, particularly within the framework of the model of the interacting electron gas in the solid state, foresees the introduction of electron basis for functions. In our case, the Bloch functions instead of the plane waves are taken as the basis of functions, i.e., wave functions of carriers are described by the dispersion law (1). The effects of electron-electron interaction causes the screening the Coulomb potential, which is found by summing the loop diagrams (random phase approximation (RPA)). In the paper [11], q-dependence of static dielectric function of the Bloch electrons with the parabolic dispersion law is described by the following model expression

$\varepsilon(\mathbf{q}, \Omega)=1+\frac{\omega_{p}^{2}}{\omega_{g}^{2}(\mathbf{q})-(\omega+i 0)^{2}}$,

where $\omega_{g}(\mathbf{q})$ is the effective $\mathbf{q}$-dependent gap frequency and $\omega_{p}$ is the plasma frequency of valence electrons.

\section{Polarization properties of the intermediate dimension structures}

In this paper, the dielectric function is calculated from the Bloch functions, considering the environment anisotropy (the dispersion law (1)). Since we deal with an electron subsystem, dielectric function should be studied depending on the direction being vector rather than a tensor.

In the RPA, $\delta(\mathbf{q}, \Omega)$ components, namely, real $\varepsilon_{1}(\mathbf{q}, \Omega)$ and imaginary $\varepsilon_{2}(\mathbf{q}, \Omega)$ ones are connected with the polarization loop $\Pi(\mathbf{q}, \Omega)[13]$ as follows:

$\varepsilon_{1}(\mathbf{q}, \Omega)=1+\operatorname{Re} \Pi(\mathbf{q}, \Omega) V_{0}(\mathbf{q})$,

$\varepsilon_{2}(\mathbf{q}, \Omega)=\operatorname{Im} \Pi(\mathbf{q}, \Omega) \quad V_{0}(\mathbf{q})$.

Here,

$$
\begin{aligned}
& \Pi(\mathbf{q}, \Omega)= \\
& =i \int G_{0}(\mathbf{k}+\mathbf{q} / 2, \omega+\Omega / 2) G_{0}(\mathbf{k}-\mathbf{q} / 2, \omega-\Omega / 2) d \mathbf{k} d \Omega .
\end{aligned}
$$

$\Pi(\mathbf{q}, \Omega)$ is the polarization loop calculated in terms of Green's functions when $T=0$ [12]:

$$
\begin{aligned}
& G_{0}(\mathbf{k}, \omega)= \\
& =\left\{\omega-E(\mathbf{k})+\varepsilon_{F}+\imath \delta \operatorname{sign}\left[E(\mathbf{k})-\varepsilon_{F}\right]\right\}^{-1} .
\end{aligned}
$$


For 1D case such polarization loop was analyzed in $[8,9]$. Integrating over the frequency by residue method, we get

$\Pi(\mathbf{q}, \Omega)=$

$=\frac{1}{2 \pi}\left[\int_{\left\{B_{3}\right\}} R_{2} d k_{x} d k_{y} d k_{z}-\int_{\left\{A_{3}\right\}} R_{1} d k_{x} d k_{y} d k_{z}\right]$,

where

$R_{\alpha}^{-1}=E\left(\mathbf{k}-\frac{\mathbf{q}}{2}\right)-E\left(\mathbf{k}+\frac{\mathbf{q}}{2}\right)+\Omega+(-1)^{\alpha} i \delta$

$(\alpha=1,2)$.

Then in the case $\Omega=0$, the real and imaginary components of $\Pi(\mathbf{q}, \Omega)$ become

$$
\begin{aligned}
& \operatorname{Re} \Pi(\mathbf{q})=\frac{1}{2 \pi} \int_{\left\{B_{3}\right\}-\left\{A_{3}\right\}}\left[2 \alpha q_{x} k_{x}+2 \alpha q_{y} k_{y}+b \sin k_{z}\right]^{-1} d k_{x} d k_{y} d k_{z} ; \\
& \operatorname{Im} \Pi(\mathbf{q})=\frac{1}{2} \int_{\left\{B_{3}\right\}+\left\{A_{3}\right\}} \delta\left(-2 \alpha q_{x} k_{x}-2 \alpha q_{y} k_{y}-b \sin k_{z}\right) d k_{x} d k_{y} d k_{z}
\end{aligned}
$$

Eqs (9) and (10) are taken in the sense of their principal values and $b=2 t \sin \left(q_{z} / 2\right)$. In the $3 \mathrm{D}$ case, the regions $\left\{A_{3}\right\}$ i $\left\{B_{3}\right\}$ are as follows:

$$
\left\{A_{3}\right\} \in\left\{\begin{array} { l } 
{ E ( \mathbf { k } - \frac { \mathbf { q } } { 2 } ) > \varepsilon _ { F } } \\
{ E ( \mathbf { k } - \frac { \mathbf { q } } { 2 } ) < \varepsilon _ { F } }
\end{array} \text { and } / \text { or } \{ B _ { 3 } \} \in \left\{\begin{array}{l}
E\left(\mathbf{k}-\frac{\mathbf{q}}{2}\right)<\varepsilon_{F} \\
E\left(\mathbf{k}-\frac{\mathbf{q}}{2}\right)>\varepsilon_{F} .
\end{array}\right.\right.
$$

Varying the free carriers concentration one can change the value of the Fermi energy. It is well known that the carriers located in the vicinity of the Fermi level play the main role in kinetics effects. The Fermi level transition to higher than the bandwidth along $k_{z}, 2 t$, makes it possible to consider at least Q2D problems in the vicinity $\varepsilon_{\mathrm{F}} \rightarrow 2 t$. We can realize the similar Q2D one by changing $t$ parameter. Hereinafter, we will consider the above-mentioned transition by changing $\varepsilon_{\mathrm{F}}$ that is, for $\varepsilon_{\mathrm{F}}<2 t$, we deal with the anisotropical nonparabolic case, and when $\varepsilon_{\mathrm{F}}>2 t$, this is a Q2D case with isoenergetic surfaces open along $O Z$ axis, and, for $\varepsilon_{\mathrm{F}}>2 t$, this is a $2 \mathrm{D}$ case. All the parameters such as $\alpha, t, \varepsilon_{\mathrm{F}}$ are given in electron-volts.

\section{Calculations of polarization loop}

Firstly, let us consider 1D case $(\alpha=0)$, for which the dispersion law (1) is as follows:

$E\left(k_{z}\right)=t\left(1-\cos k_{z}\right)$.

In this case, the disperse dependence of real and imaginary components $\Pi\left(q_{z}, \Omega\right)$ are written in the following manner:

$$
\begin{aligned}
& \operatorname{Re} \Pi\left(q_{z}, \Omega\right)=-\frac{1}{2 \pi} \int_{\{B\}-\{A\}}\left(\Omega-b \sin k_{z}\right)^{-1} d k_{z}, \\
& \operatorname{Im} \Pi\left(q_{z}, \Omega\right)=\frac{1}{2} \int_{\{B\}+\{A\}} \delta\left(\Omega-b \sin k_{z}\right) d k_{z} .
\end{aligned}
$$

The condition (11) gives us the nonzero values of the polarization loop (13) and (14). The regions of integration are given in Fig. 1.

1. Region $|Q|>1$ where $Q=\frac{t-\varepsilon_{F}}{t}$, then $\{A\},\{B\} \in\{0\}$ or according to (5) $\Pi\left(q_{z}\right)=0$.

2. Region $R \leq Q \leq 1$ : then $\{A\} \in\left[z_{3}, z_{0}\right],\{B\} \in\left[z_{4}, z_{1}\right]$ (Fig. 1a), where $z_{3}, z_{4}$ are the roots of the equation $Q=\cos \left(z+\frac{q_{z}}{2}\right)$, and $z_{0}, z_{1}$ are the roots of the equation $Q=\cos \left(z-\frac{q_{z}}{2}\right)$.

3. Region $-R \leq Q \leq R \quad: \quad\{A\} \in\left[z_{1}^{\prime}, z_{0}^{\prime}\right],\{B\} \in\left[z_{4}^{\prime}, z_{3}^{\prime}\right]$ (Fig. 1b).

4. Region $-1 \leq Q \leq-R:\{A\} \in\left[z_{1}^{\prime \prime}, z_{4}^{\prime \prime}\right],\{B\} \in\left[z_{0}^{\prime \prime}, z_{3}^{\prime \prime}\right]$

(Fig. 1c).

The integrals (13) and (14) can be found analytically. In the static case $(\Omega=0)$, it is

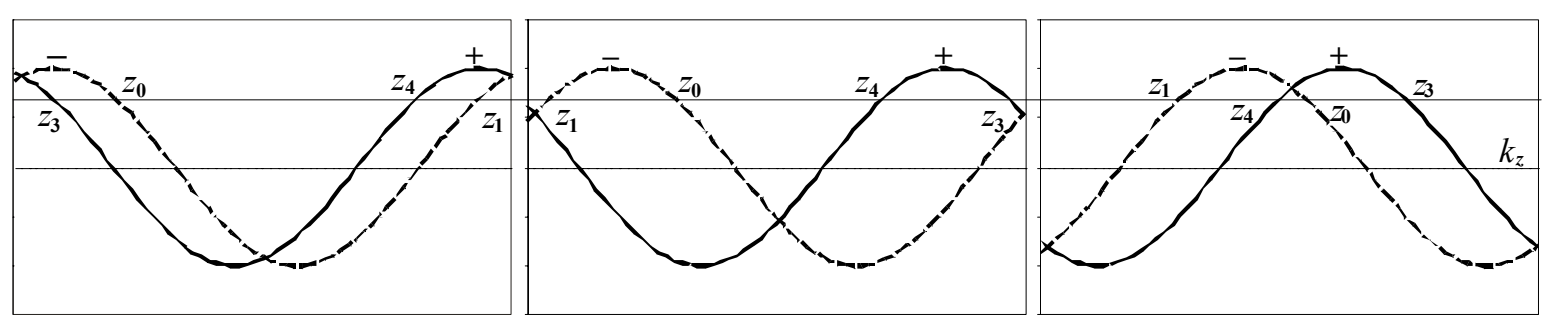

a c

Fig. 1. The regions of integration $\{A\},\{B\}$ according to (11) in the 1D case. 
$\operatorname{Re} \Pi\left(q_{z}\right)=\frac{1}{2 \pi t \sin \frac{q_{z}}{2}} \ln \left|\frac{\operatorname{tg} \frac{\widetilde{z}_{1}}{2}}{\operatorname{tg} \frac{\widetilde{z}_{0}}{2}}\right|$.

The limits of integration are determined from the condition (6) (Fig. 1), and they are different depending on $\mathrm{Q}$ value [8]. They can be compared with $\operatorname{Re} \Pi\left(q_{z}\right)$ for the $1 \mathrm{D}$ case with the parabolic dispersion law [12]

$$
\operatorname{Re} \Pi\left(q_{z}\right)=N_{1 D}(0) \frac{2 k_{F}}{q_{z}} \ln \left|\frac{q_{z}+2 k_{F}}{q_{z}-2 k_{F}}\right| .
$$

As seen from (16), the polarization loop has singuliarity in one point $q_{z}=2 k_{\mathrm{F}}$ while, for our case, it has an additional point $q_{z}=2\left(\pi-k_{\mathrm{F}}\right)$ (see Fig. 1 in [8]).

In the case of $3 \mathrm{D}$ anisotropy $(\alpha \neq 0, t \neq 0)$, the similar calculations of $\{A\},\{B\}$ regions and $\operatorname{Re} \Pi(\mathbf{q}), \operatorname{Im} \Pi(\mathbf{q})$ were performed numerically. They showed that the behaviour of the polarization loop does not change qualitively at the transition from $1 \mathrm{D}$-system to $3 \mathrm{D}$ anisotropical one for given band filling (Fermi level), i.e., the quantity of singuliarity points does not decrease, while the $\operatorname{Re} \Pi\left(q_{z}\right), \operatorname{Im} \Pi\left(q_{z}\right)$ values increase. The further increase of band filling, i.e., the transition from 3D-case to Q2D-one, causes the decrease of the quantity of singuliarity points and an increase of $\operatorname{Re} \Pi\left(q_{z}\right), \operatorname{Im} \Pi\left(q_{z}\right)$ values (Figs 2, 3).

\section{The potential screened by interelectron interaction}

The obtained disperse dependences for the components of dielectric function were used for the $\widetilde{V}(\vec{q})$ calculations according to (2). Here, the Coulomb potential $V_{0}(\vec{q})$ is taken from (3), i.e., the potential used for 3D-case. Such a choice is caused by the above-mentioned purpose of

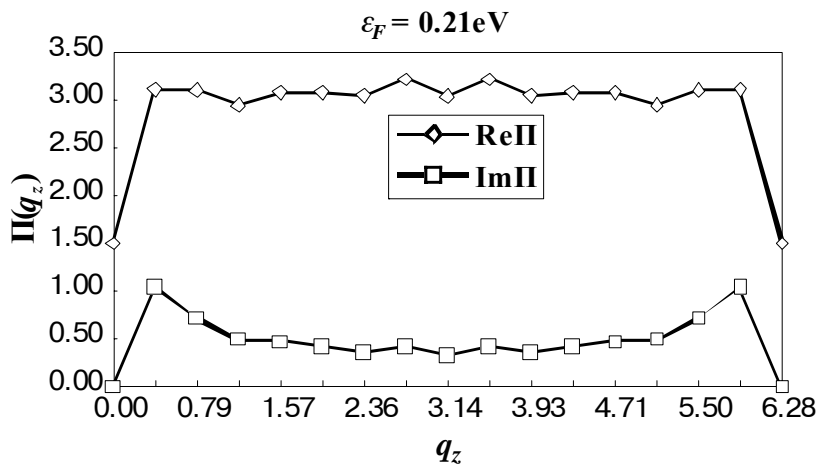

Fig. 3. Dependence of the polarization loop $\operatorname{Re} \Pi\left(q_{z}, 0,0\right)$, $\operatorname{Im} \Pi\left(q_{z}, 0,0\right)$ for the Q2D case $\left(\varepsilon_{F}=0.21 \mathrm{eV}\right)$.

the paper which lies in studying the screening effects in the structures of intermediate dimension. These effects are caused by strongly anisotropic but still 3D-structure. That is why the coordinate dependence of $\tilde{V}(r)$

$\widetilde{V}(r)=\int_{-\pi}^{\pi} \widetilde{V}(\mathrm{q}) \cos \mathrm{qr} d q_{x} d q_{y} d q_{z}$

is considered as $3 \mathrm{D}$ integral.

Let us analyze the $\widetilde{V}(r)$ dependence on the coordinates in the directions (001) and (111). In the (001) case, $\widetilde{V}(r)$ dependence for different $\varepsilon_{\mathrm{F}}$ is represented in Fig. 4. As seen from Fig. 4, the amplitude oscillations (curve 1) take place at smaller distances than that for isotropic Fermi-gas (insertion in Fig. 4). This difference is evidently caused by the nonparabolic dispersion law. The decrease of $\widetilde{V}(r)$ (curves 1-3), i.e., at the transition from quasi 2D to $3 \mathrm{D}$ anisotropic case, causes a decrease of the first minimum in $\widetilde{V}(r)$ dependence just to negative values and shifts them in

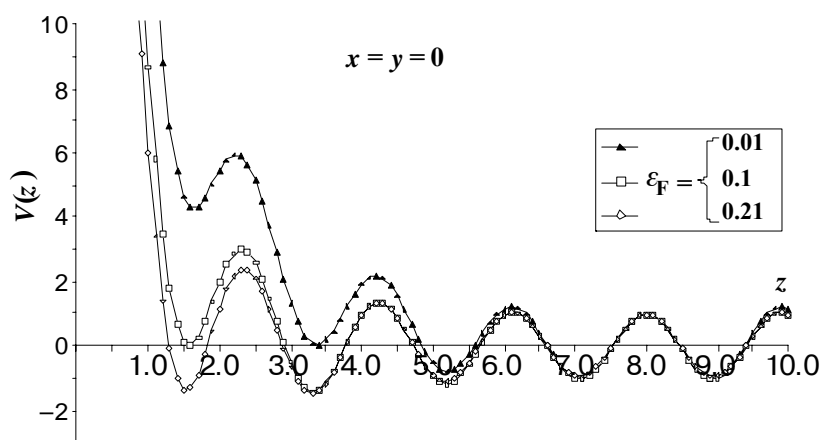

Fig. 4. Dependence of $\tilde{V}(z)$ in the direction (001) for different $\varepsilon_{F}$ values.
Fig. 2. Dependence of the polarization loop $\operatorname{Re} \Pi\left(q_{z}, 0,0\right)$, $\operatorname{Im} \Pi\left(q_{z}, 0,0\right)$ for the $3 \mathrm{D}$ anisotropic case $\left(\varepsilon_{F}=0.01 \mathrm{eV}\right)$.

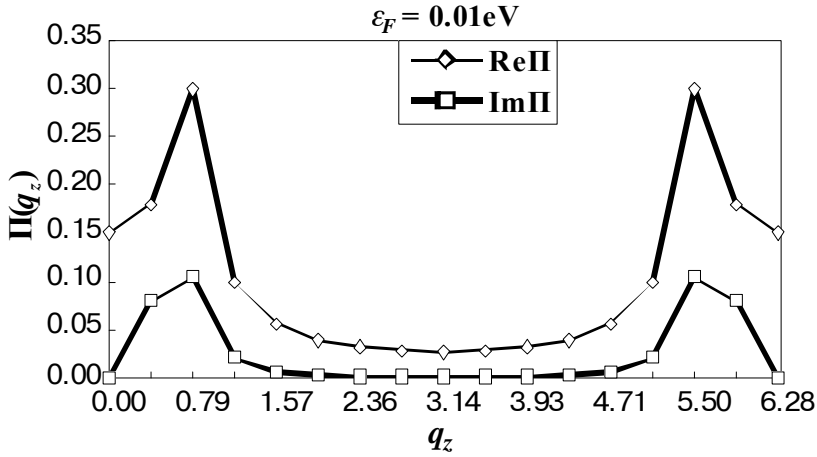

(C) 2005, V. Lashkaryov Institute of Semiconductor Physıcs, svaıvnaı Acauemy of scıences of ukraıne 


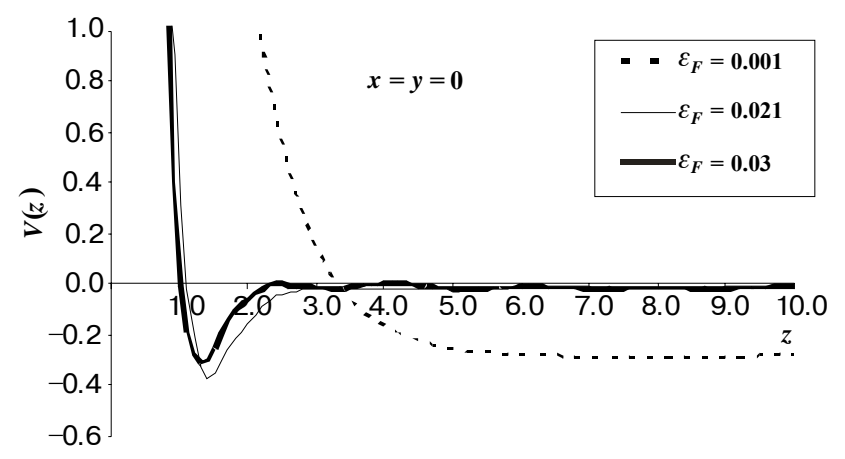

Fig. 5. Dependence of $\widetilde{V}(z)$ in the direction (111) for different $\varepsilon_{F}$ values.

the direction of smaller $z$-distances. In the vicinity of $\varepsilon_{F}=2 t$, electrons at distances $z \sim 1.5 a_{0}\left(a_{0}\right.$ is the lattice constant) are most attracted, while when $\widetilde{V}(r)$ amplitude oscillations practically do not depend on $\varepsilon_{F}$.

$\widetilde{V}(r)$ dependence along (111) direction looks somewhat different: the first minimum in the $z \sim 1.5 a_{0}$ vicinity (Fig. 5a) disappears and oscillations in the coordinate dependence are noticeable only after 15 -fold extension.

The coordinate $\tilde{V}(r)$ dependence in (111) direction compared with that in the direction (001) changes as follows: i) when $\varepsilon_{F}=0.01$, the oscillation period significantly increases, while the first local minimum (typical for (001) direction) vanishes; ii) when $\varepsilon_{\mathrm{F}}=0.21$, the oscillation period also increases significantly, and the depth of the first minimum decreases.

\section{Conclusions}

Polarization properties of the electron subsystem in the structures of intermediate dimension, in particular, in the Q2D structures depending on the degree of quasi-twodimensionality are studied. The peculiarities of their electron band structure are characterized by the model dispersion law.

The analysis of the polarization properties of electron subsystem in the Q2D structures within the framework of the RPA shows:
1. When changing the degree of Q2D, the number of singuliarity points in the disperse behaviour of the polarization loop $\Pi\left(q_{z}\right)$ and dielectric function in the $k_{z}$ direction do not change while its number changes inside the $X O Y$ plane.

2. The character of alternating oscillations of the potential screened by interelectron interaction is stronger pronounced as compared to the isotropic crystal structures. This is caused by nonparabolic effects.

Thus, changing the degree of quasi-two-dimensionality of the layer crystal by intercalation, stage ordering, hydrostatic or axial pressure makes it possible to significantly affect the properties of the electron subsystem. That is why we should take into consideration the screening effects analyzing the phenomena that take place in layer crystals.

\section{References}

1. L. Gordstein, F. Glas, J.Y. Marzin et al. // Appl. Phys. Lett. 47, p.1099 (1985).

2. V.M. Ustinov // Physics and Technique of Semiconductors 38, N8, p. 963 (2004) (in Russian).

3. G.B. Ibragimov // J. Phys.: Condens Matter 14, p. 8145 (2002).

4. B. Vinter and L. Thibaudean, Bound to free state infrared absorption and selection rules in quantum wells. Intersubband transition in quantum wells, Ed. by E. Rosencher, B. Vinter, B. Levine, New York (1992).

5. H.N. Spector // Phys. Rev. B 28, p. 971 (1983).

6. I.I. Grigorchak // Phys. Chem. Solid State 2, N1, p. 7 (2003).

7. R.F. Fivaz // J. Phys. Chem. Solids 28, N4, p. 839 (1967).

8. B. Lukiyanets, N. Tovstyuk // Condensed Matter Phys. 8, p. 99 (1996).

9. B.A. Lukiyanets, N.K. Tovstyuk // Zhurn. Fiz. Doslidgen' 1, p. 251 (1997).

10. N. Ashcroft, N. Mermin, Solid State Physics, Mir, Moscow (1979)(in Russian).

11. F. Bechstedt et al. // Phys. status solidi (b) 126, p. 575 (1984).

12. D. Pines, The many-body problem, W.A. Benjamin Inc., New York (1961).

13. H.I. Starnberg, H.E. Brauer, H.P. Hughes // Phys. and Chem. of Materials with Low-Dimensional Structures 24, p. 41 (2000). 